\title{
ROUGH FRACTIONAL INTEGRAL OPERATORS AND BEYOND ADAMS INEQUALITIES
}

\section{Daniel Salim, Yudi Soeharyadi and Wono Setya Budhi}

Abstract. We consider the boundedness of fractional integral operators with rough kernel from Morrey spaces $L^{p, \lambda}$ to $L^{q, \mu}$. Our main concern is proving the boundedness property for $\mu<\lambda$ as an extension of Adams inequality on some special subsets of the operator's domain namely classes of $A_{p}$, simple function, and radial function respectively. For radial function, we prove the boundedness on local Morrey spaces. We also prove the boundedness property for $\mu \geqslant \lambda$ as well as the special case of $q \leqslant p$. It is interesting on its own term since the operator is not bounded from $L^{p}$ to $L^{q}$ if $q \leqslant p$. We also establish necessary conditions for boundedness. Our proposed condition for boundedness includes the sufficient conditions for both Adams inequality and Spanne inequality.

Mathematics subject classification (2010): 42B20, 42B25.

Keywords and phrases: Fractional integral operator, Rough kernel, Morrey spaces, Adams inequality, Spanne inequality.

\section{REFERENCES}

[1] B. Muckenhoupt And R. L. Wheeden, Weighted norm inequalities for singular and fractional integrals, T. Am. Math. Soc. 161, (1971), 249-258.

[2] D. R. Adams, A note on Riesz potentials, Duke Math. J. 42, 4 (1975), 765-778.

[3] H. Gunawan, D. I. HAKIM, AND M. IDRIS, Proper inclusions of Morrey spaces, Glas. Mat. 53, 1 (2018), 143-151.

[4] J. DuOAndikoetxea, Fourier analysis, American Mathematical Soc, vol. 29, 2001.

[5] J. DuOANDIKOETXEA, Fractional integrals on radial functions with applications to weighted inequalities, Ann. Mat. Pura Appl. 192, 4 (2013), 553-568.

[6] J. PeETRE, On the theory of $L^{p, \lambda}$ spaces, J. Funct. Anal. 4, 1 (1969), 71-87.

[7] P. A. OlsEn, Fractional integration, Morrey spaces and a Schrödinger equation, Commun. Part. Diff. Eq. 20, 11-12 (1995), 2005-2055.

[8] T. IIDA, Weighted inequalities on Morrey spaces for linear and multilinear fractional integrals with homogeneous kernels, Taiwan J. Math. 18, 1 (2014), 147-185.

[9] Y. Komori-Furuya AND E. S ATO, Fractional integral operators on central Morrey spaces, Math. Inequal. Appl 20, 3 (2017), 801-813.

[10] Y. SAWANO, S. SugANO, AND H. TANAKA, Generalized fractional integral operators and fractional maximal operators in the framework of Morrey spaces, T. Am. Math. Soc. 363, 12 (2011), 6481-6503. 